\title{
Supervisión y Control Industrial a través de Teléfonos Inteligentes usando un Computador de Placa Única Raspberry Pi
}

\author{
Sergio A. Castro(1), Byron Medina ${ }^{(1)}$ y Luis L. Camargo(2) \\ (1) Universidad Francisco de Paula Santander, Grupo de Investigación y Desarrollo en \\ Telecomunicaciones, GIDT. Avenida Gran Colombia No. 12E-96, San José de Cúcuta, Colombia. \\ (e-mail: sergio.castroc@ufps.edu.co; byronmedina@ufps.edu.co) \\ (2) Universidad del Magdalena, Grupo de Investigación en Desarrollo Electrónico y Aplicaciones Móviles, \\ GIDEAM. Carrera 32 No. 22-08, Santa Marta, Colombia. (e-mail: Icamargoa@unimagdalena.edu.co)
}

Recibido Ago. 24, 2015; Aceptado Oct. 28, 2015; Versión final Dic. 14, 2015, Publicado Abr. 2016

\begin{abstract}
Resumen
El artículo presenta el desarrollo e implementación de una aplicación para teléfonos inteligentes, que supervisa y controla un proceso industrial a través del sistema embebido Raspberry, conectado a Internet mediante el protocolo de intérprete de órdenes seguras. El sistema integra las ventajas de la instrumentación virtual y la movilidad, con el objetivo de mejorar los procesos de producción y la competitividad de las empresas. La aplicación fue elaborada con la metodología para el desarrollo de aplicaciones móviles, que contempla las fases de análisis, diseño, desarrollo, pruebas de funcionamiento y entrega. Además, la aplicación fue utilizada en una planta piloto para verificar su correcto funcionamiento y rendimiento en un entorno real para evaluar su usabilidad. Se concluye que la aplicación tiene potencial de éxito para ser implementada a gran escala, siendo de fácil manipulación, atractiva y eficiente.
\end{abstract}

Palabras clave: control industrial; automatización; internet; Raspberry; computador de placa única; usabilidad de software

\section{Supervision and Industrial Control through Smartphone using a Single-board Computer Raspberry Pi}

\begin{abstract}
The paper presents the development and implementation of an application for smartphones, which monitors and controls a real-time industrial process through an embedded system based on Raspberry, connected to the Internet using the Secure Shell protocol. The system integrates the advantages of virtual instrumentation with mobility for optimizing production processes and competitiveness in enterprises. The app was done using the mobile app development methodology, which consists of five stages: analysis, design, development, performing testing and delivery. Furthermore, the app was used in a pilot plant to verify its adequate operation and performance in a real environment, to evaluate its usability. It is concluded that the app has potential success for being implemented on a large scale, being easy to handle, attractive and efficient.
\end{abstract}

Keywords: industrial control; automation; internet; Raspberry; single-board computer; software usability 


\section{INTRODUCCIÓN}

Según el documento de la transformación de las telecomunicaciones (UIT, 2015), las telecomunicaciones, la tecnología de la información (TI) y la informática en el transcurrir del tiempo, han incrementado su convergencia, robustez y versatilidad. Los dispositivos conectados son, sin lugar a dudas, portátiles, pequeños y más numerosos. En un futuro las personas disfrutarán de una conectividad más rápida mientras se desplazan, y estarán permanentemente conectadas a diferentes redes en todas partes, en todo momento y a través de cualquier dispositivo, gracias a la denominada conectividad ubicua. Para el 2018 se espera que los teléfonos inteligentes en América Latina alcancen una penetración del 60 por ciento, incrementando el uso de datos móviles y posicionándose como el principal motor de crecimiento de los ingresos de venta de telecomunicaciones para la región (lacopino, 2014). De igual manera, se proyecta que el tráfico de datos móviles de teléfonos inteligentes en América Latina, crecerá 12 veces desde 2014 hasta 2019, con una tasa de crecimiento anual compuesta del 64 por ciento; alcanzando el 46,6 por ciento de las conexiones de dispositivos en 2019, y el 64,4 por ciento del tráfico (Cisco, 2015).

Se ha identificado que la innovación, apoyada en los avances científicos y tecnológicos involucrados a la producción, desarrolla o mejora los productos y procesos existentes. La innovación promueve el crecimiento de la productividad y la competitividad de las empresas, características fundamentales en un mundo globalizado de permanente cambio, reformulando la dinámica de los negocios (Rodríguez, 2014). Sin embargo en América Latina son escasas las Pequeñas y Medianas Empresas (PYMES) que cuentan con una estrategia de desarrollo y consolidación fundamentada en la innovación y, si la tienen, normalmente responden a mecanismos informales y no al resultado de una rigurosa planificación (OCDE/CEPAL, 2012).

Avanzar hacia un escenario de crecimiento empresarial sostenible con mayor igualdad supone construir mecanismos que permitan densificar la matriz productiva, diversificarla en términos de actividades de alta productividad e incorporar en ellas conocimiento. La fuerza motriz de esa transformación es la generación de conocimiento y la promoción de innovaciones y nuevas tecnologías a lo largo del sistema productivo. En el corazón de ese proceso se encuentran las Tecnologías de la Información y las Comunicaciones (TIC) y la difusión del paradigma digital. Esta disyuntiva es fundamental para definir las políticas tecnológicas y de sociedad de la información (CEPAL, 2013), y mejorar los procesos, productos y servicios. La automatización de los procesos es un factor importante para la transformación de las empresas, ésta integra conocimientos de diseño mecánico, ciencias de la computación, desarrollo de hardware, ingeniería de control y redes de comunicación; haciendo uso de tecnologías modernas para convertir la industria en sistemas productivos, óptimos y eficientes (Breivold et al., 2013).

América Latina y el Caribe han avanzado hacia la sociedad de la información, la mayoría de los países de la región han formulado recientemente importantes políticas TIC nacionales y sectoriales, logrando experiencias muy interesantes de inclusión digital. Los resultados alcanzados por el acceso a los medios de comunicación móviles y a Internet han contribuido a mejorar las condiciones de vida de la mayoría de la población de la región, a modernizar la gestión pública y, aunque en forma muy heterogénea, a elevar la eficiencia empresarial (CEPAL, 2013). De acuerdo con lo anterior, el proyecto promueve la creación de capacidades en las TIC para las Micro, Pequeñas y Medianas Empresas (MIPYMES) de la región, mediante el desarrollo de una aplicación móvil para teléfonos inteligentes, que permite a un operario supervisar y controlar un proceso industrial. Además, demuestra que es posible incorporar a los teléfonos móviles como herramienta industrial, agregando el atributo de movilidad en la tradicional instrumentación virtual.

La iniciativa en desarrollo OPC UA (OLE for Process Control Unified Architecture) es una propuesta de la Fundación OPC para la transmisión segura, fiable y neutra con respecto a los datos en la planta y en la información pre procesada de los niveles de producción y, de los sistemas de planificación de producción (Manufacturing Execution System, MES) y de empresa (Enterprise Resource Planning, ERP). Es decir, constituye la unión entre los sistemas de manufactura y planificación empresarial, y los equipos embebidos de automatización de plataforma independiente como la Raspberry Pi, con sistema operativo Linux (OPC Foundation, 2015).

En la actualidad existen Apps en la Play Store, que permiten controlar mediante consola de comandos sistemas embebidos como Raspberry, Arduino y Odroid. Aplicaciones como ConnectBot, RaspController, Odroid app y Arduino Total Controll, entre otras. Estas aplicaciones permiten manipular los puertos de propósito general de estos dispositivos mediante el teléfono. También hay disponible aplicaciones de Supervisión, Control y Adquisición de Datos (Supervisory Control And Data Acquisition, SCADA), de los principales fabricantes de equipos de automatización basadas en OPC (OLE for Process Control) estándar de comunicación en el campo del control y la supervisión de procesos industriales (Google, 2015). 
La aplicación elaborada, a diferencia de las mencionadas, permite la supervisión y el control de un proceso industrial mediante la comunicación con equipos embebidos de automatización, de plataforma independiente y de código abierto, conectados a la red Internet, como la Raspberry Pi.

La Raspberry $\mathrm{Pi}$ es un ordenador de placa reducida de la fundación Raspberry, con sistema integrado, unidad central de procesamiento, memoria RAM, almacenamiento integrado, puertos de propósito general, salidas multimedia, periféricos de bajo nivel y conectividad de red ethernet. Este sistema embebido puede actuar como controlador y atender peticiones de aplicaciones móviles con sistema operativo Android. La Raspberry Pi y la aplicación móvil Android, son los componentes tecnológicos principales de la propuesta.

El artículo describe el desarrollo e implementación de la aplicación móvil en el sistema de supervisión y control, como solución tecnológica que se incorpora para gestionar el proceso; además se detallan las fases de la metodología utilizada en el desarrollo, y los resultados de las pruebas de funcionamiento mediante una herramienta computacional, equipos móviles celulares reales y las técnicas de evaluación de las 6M's (Ahonen et al., 2002) y la usabilidad (Mascheroni et al., 2013; Hurtado et al., 2013; Alarcón et al., 2014), emitiendo conceptos operativos. Finalmente se presentan las conclusiones del proyecto.

\section{METODOLOGÍA}

La aplicación móvil se elaboró implementando la Metodología para el Desarrollo de Aplicaciones Móviles (Gasca et al., 2014), que se basa en la experiencia en el desarrollo de aplicaciones móviles (Camargo et al., 2013; Rivera et al., 2014; Medina et al., 2015), la evaluación del potencial de éxito para servicios de tercera generación denominada 6M's (Ahonen et al., 2002), la evaluación de usabilidad del software (Mascheroni et al., 2013; Hurtado et al., 2013; Alarcón et al., 2014), y principalmente en los valores de las metodologías ágiles (Beck et al., 2001). La metodología define cinco fases llamadas análisis, diseño, desarrollo, pruebas de funcionamiento y entrega.

\section{Análisis}

En la fase de análisis se definieron las características del entorno de la aplicación, como la obtención y clasificación de los requerimientos y la personalización del servicio. La obtención de requerimientos determinó una aplicación que permitió al usuario ejercer un control del proceso industrial de estampado de piezas, en modo manual o automático. Además, la aplicación agregó un modo de referencia para suministrar al usuario la información que lo orientó en la interacción con la interfaz.

La clasificación de requerimientos definió las características de software del dispositivo móvil como el sistema operativo Android 2.0 o superiores, el lenguaje de programación Java (Oracle, 2014) y el Entorno de Desarrollo Integrado (Integrated Development Environment, IDE) Eclipse versión Kepler (Eclipse, 2014), con el conjunto de herramientas para el desarrollo de software (Software Development Kit, SDK) y el kit de desarrollo de aplicaciones para Android (Android Developers Tools, ADT) (Android, 2014). De igual manera, se definieron las características de software del sistema embebido Raspberry $\mathrm{Pi}$ B, como el sistema operativo Raspbyan (New Out Of the Box Software, NOOBS) (Raspberry, 2014), y las librerías WeblOPi (Webiopi, 2014), la Wiring Pi (Wiring Pi, 2014) y la Pi4J (Pi4J, 2014). La personalización del servicio determinó particularidades de la aplicación como la comunicación a través del Protocolo de Control de Transmisión/Protocolo de Internet (Transmission Control Protocol/Internet Protocol, TCP/IP) con el sistema embebido y, la implementación de actividades para identificar el usuario, establecer la conexión, y emular e interactuar gráficamente con el proceso industrial.

\section{Diseño}

En la fase de diseño, utilizando el IDE Eclipse, se representó la aplicación mediante el Lenguaje Unificado de Modelado (Unified Modeling Language, UML) para sistemas de software, a través del diagrama de casos de uso y el diagrama de clases, que soportaron el funcionamiento de las actividades de la aplicación. En la figura 1, se define el diagrama de casos de uso que indica las acciones que puede realizar el usuario en la interacción con la aplicación. El usuario accede a la bienvenida representada por el caso Master_home, luego accede a la aplicación mediante el caso Master_login y al controlador del sistema mediante el caso Master_access. Después de acceder a la aplicación y al controlador, el usuario puede instalar librerías usando Master_install, y finalmente acceder al control de las variables del proceso industrial a través del caso Master_mobile. 


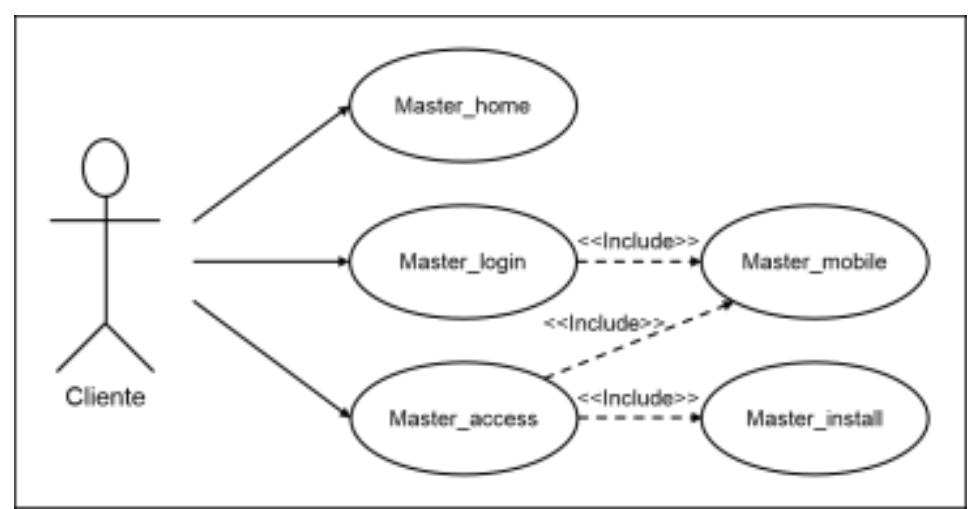

Fig. 1: Diagrama de casos de uso de la aplicación móvil

La aplicación móvil se denominó MasterMobile y está compuesta por cinco clases primarias (Master_home, Master_login, Master_access, Master_install y Master_mobile) y cinco clases secundarias (auto, gpio, login, tareain y tareain2). Las clases primarias tienen esta denominación porque asocian una actividad de igual nombre, mientras que las clases secundarias no tienen asociada una actividad y apoyan los procesos internos de la aplicación, como el control del puerto de propósito general del sistema embebido, el direccionamiento para la instalación de librerías, la validación de la lógica de control y la representación del proceso industrial mediante imágenes. El diagrama de clases identifica el funcionamiento y correlación entre las clases de la aplicación móvil Android, ver figura 2.

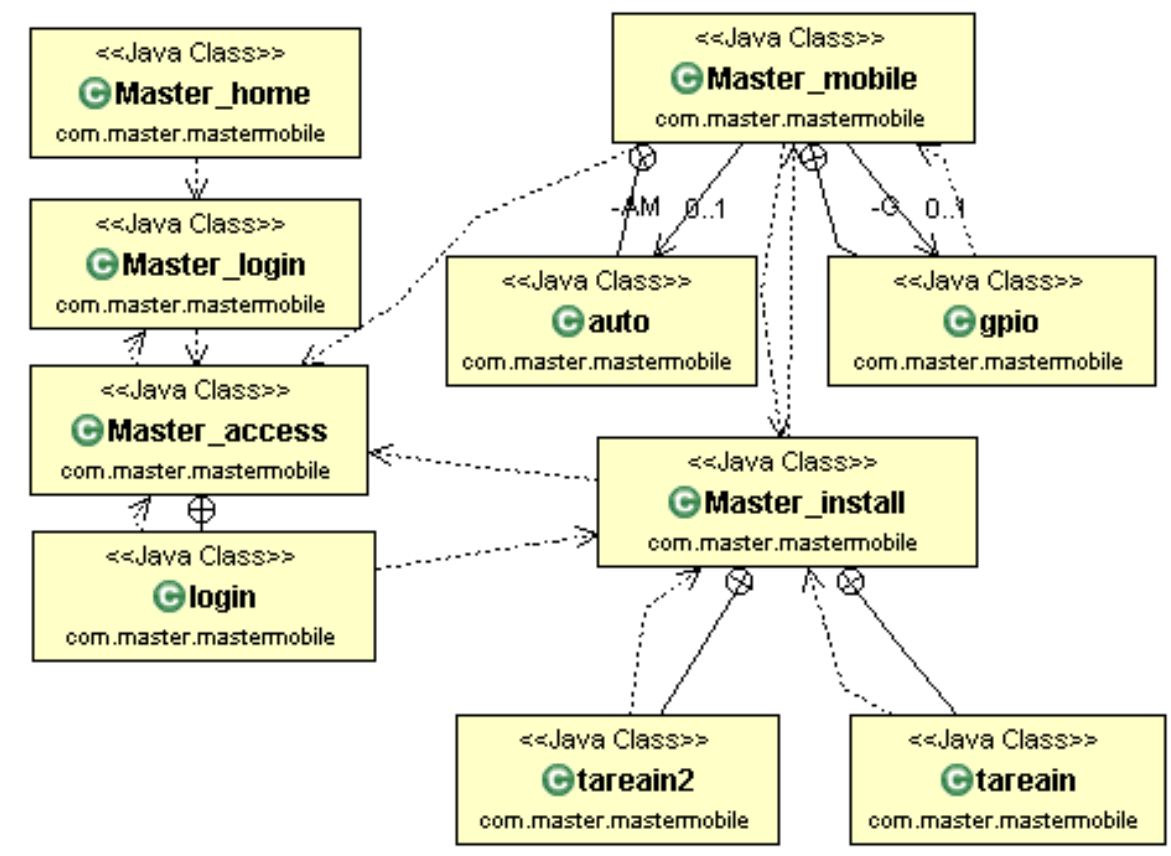

Fig. 2: Diagrama de clases de la aplicación móvil

La aplicación inicia en la clase de bienvenida (Master_home), que se enlaza con la clase de identificación de usuario (Master_login) para comprobar los atributos de acceso; luego continúa a la clase para acceder al sistema (Master_access) que según la validación de variables, permite continuar a la clase de acciones iniciales de instalación dentro del sistema embebido (Master_install), o devolverse a la clase Master_login. Posteriormente, la clase Master_install permite ir a las clases de instalación de las librerías para el sistema embebido (tareain y tareain2), o pasar a la clase encargada de supervisar y controlar el proceso industrial (Master_mobile). Lo anterior, depende de la comprobación de parámetros y atributos dentro de la clase. Finalmente, la clase Master_mobile según la validación de condiciones, permite el acceso a las clases referentes a los modos de operación, como la clase auto para el modo automático o la clase gpio para el modo manual.

\section{Desarrollo}

En la fase de desarrollo de la aplicación móvil, se implementó el diseño mediante la codificación del diagrama de clases, las pruebas unitarias, la documentación del código y la codificación de las ayudas; utilizando el IDE Eclipse. El diagrama de clases se codificó en el lenguaje de programación Java; las 
pruebas unitarias comprobaron la correcta operación de los elementos, de manera individual y en conjunto, al comparar los resultados obtenidos con los esperados; se documentó el código en el proceso de codificación y prueba de los elementos; y finalmente se codificaron las ayudas mediante el manual de instalación y de usuario, y la interfaz de la aplicación en el lenguaje Java.

La aplicación móvil elaborada hace parte de un proceso industrial, éste además está compuesto por la planta, el controlador y los sensores. La planta es un sistema neumático secuencial industrial. El controlador es el sistema embebido Raspberry Pi B, y es quien permite la adquisición y manipulación de los datos. Los sensores comprueban el estado de la variable controlada. La aplicación móvil es una interfaz de usuario que permite gestionar desde un teléfono inteligente el estado de las variables de la planta. En la figura 3 se ilustra la implementación del proyecto.

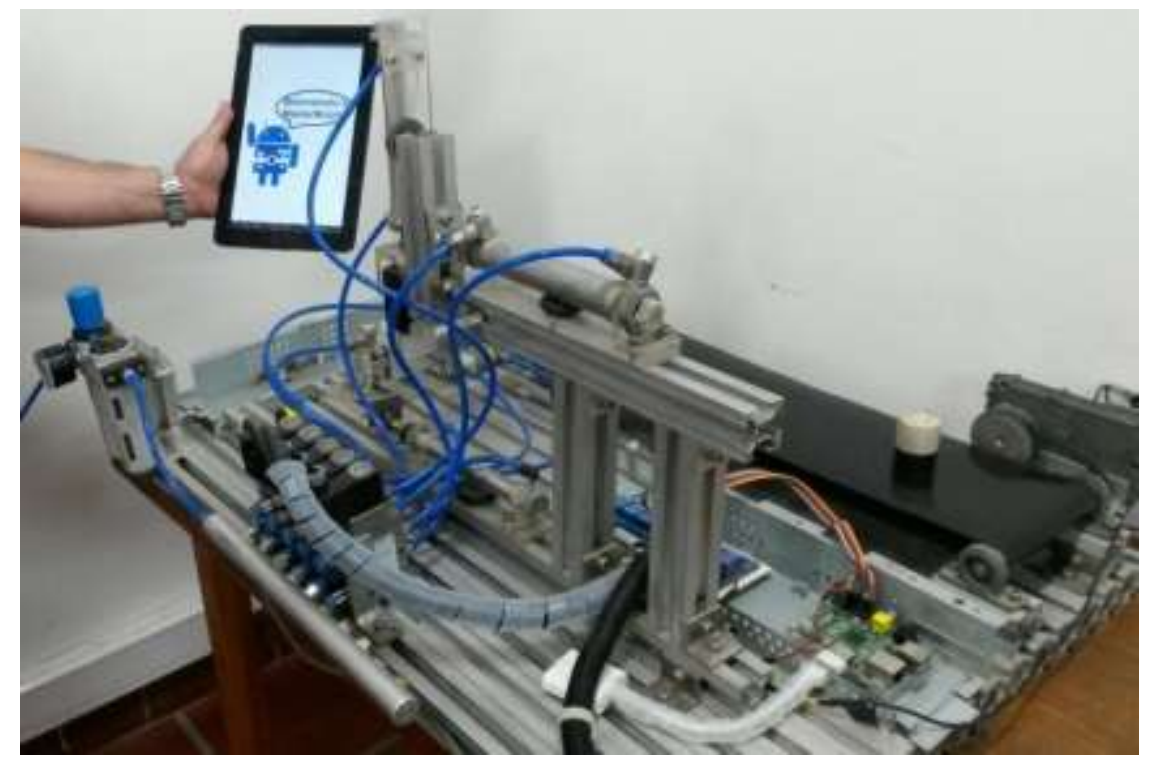

Fig. 3: Implementación del proceso industrial

Los componentes de la arquitectura de red del sistema desarrollado, se detallan en la figura 4, como son el Equipo de Usuario (User Equipment, UE), la infraestructura de red del operador de telefonía móvil celular (Agusti et al., 2010), la Red de Área Local (Local Area Network, LAN) y el sistema de control. Al lado izquierdo superior de la figura se observa el UE interactuando con la infraestructura de red del operador de telefonía móvil celular, la cual se divide de forma lógica en una infraestructura de Red de Acceso (Access Network, AN) y una de Red Troncal (Core Network, CN); esta infraestructura interconecta el UE con Internet. En la parte inferior se encuentran el sistema de control, compuesto por la planta, el controlador y los sensores; la Raspberry está conectada a un conmutador, quien a su vez se conecta con un enrutador IP convencional a Internet. Adicionalmente, el UE puede conectarse con el conmutador a través de la LAN ubicada en la industria.

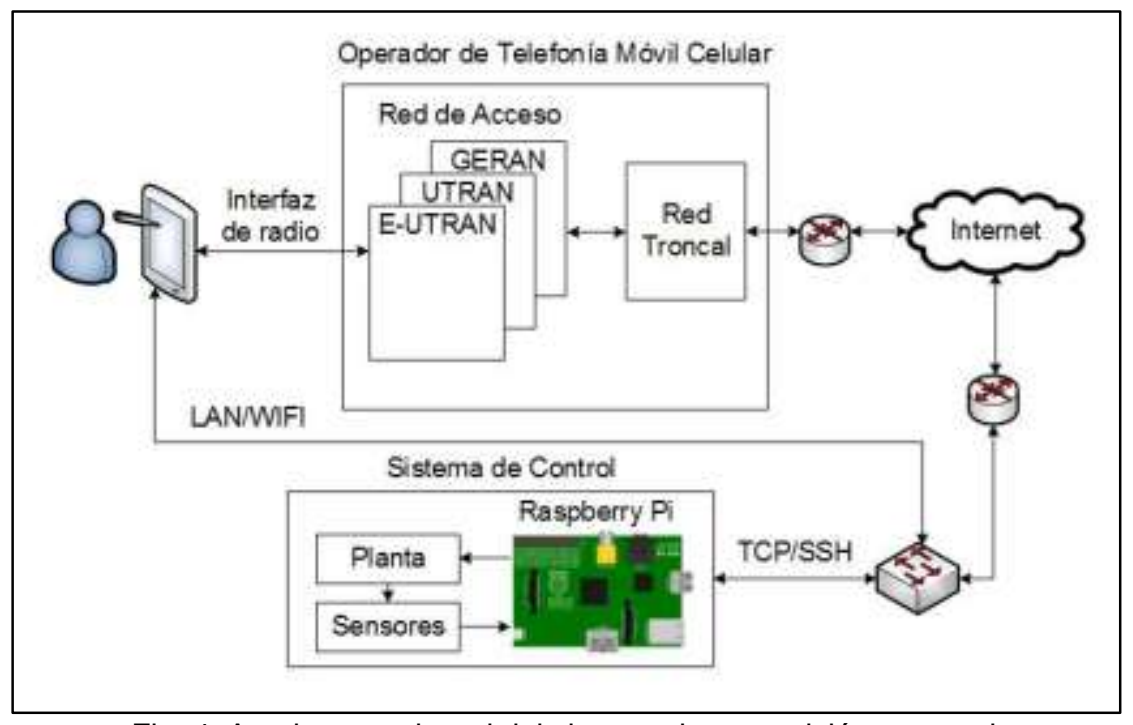

Fig. 4: Arquitectura de red del sistema de supervisión y control 
La arquitectura permite la transferencia de datos entre el UE y la Raspberry, utilizando el Protocolo de Control de Transmisión (Transmission Control Protocol, TCP), y el protocolo de intérprete de órdenes seguras (Secure SHell, SSH). Es decir, la arquitectura de red implementada, comunicó al UE con la Raspberry, haciendo uso de la interfaz de radio del operador de telefonía móvil o de la red privada de la planta, este intercambio de datos permitió la supervisión y el control del estado de las variables de la planta desde el teléfono inteligente, de manera remota.

\section{Pruebas de funcionamiento}

En esta fase se verificó el funcionamiento de la aplicación móvil de tres maneras, utilizando una herramienta de simulación, usando dos teléfonos móviles celulares, y aplicando dos técnicas de evaluación denominadas usabilidad y 6M's. Las pruebas de funcionamiento se exponen en la sección de resultados.

\section{Entrega}

En esta fase se entregaron el ejecutable de la aplicación móvil, el código fuente, la documentación con la metodología implementada en el desarrollo de la aplicación, y los manuales de instalación del sistema y del usuario. Los manuales establecen el proceso de instalación, el actuar ante posibles fallas en la ejecución, y los requerimientos técnicos de hardware y software del teléfono inteligente, para la operación óptima de la aplicación móvil. La distribución del apk en la jornada de evaluación, se realizó a través de Internet en el siguiente enlace https://www.dropbox.com/sh/haoim5allvye4uf/AAAuarFxsDvCP-8j8oUMVCjha?dl=0.

\section{RESULTADOS Y DISCUSIÓN}

A continuación se enuncian los resultados relevantes de evaluar la aplicación móvil en la fase de pruebas de funcionamiento. La herramienta de simulación utilizada fue el kit de desarrollo para Android apoyado en Eclipse, que implementa un emulador virtual de dispositivo móvil Android; este entorno se usó para verificar el funcionamiento de cada actividad sin necesidad de instalar la aplicación en el dispositivo móvil, y permitió fácilmente la corrección de errores. En la figura 5, se muestra el emulador del entorno de desarrollo.

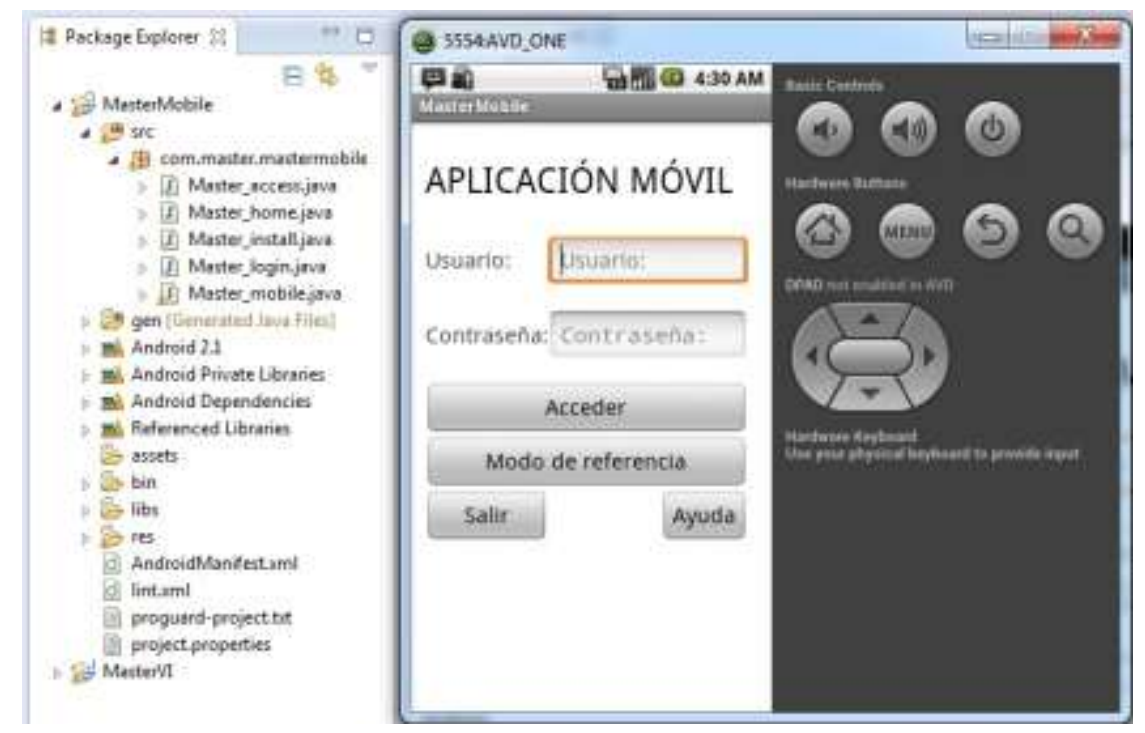

Fig. 5: Emulador del entorno de desarrollo eclipse

En la herramienta se configuró el emulador virtual para dispositivos Android (Android Virtual Device, AVD), basado en las características técnicas del dispositivo Samsung Galaxy SIV mini y Moto G, como las dimensiones de la pantalla, la versión del sistema operativo, la capacidad de almacenamiento, entre otras. Con el emulador, se verificó el correcto funcionamiento de las diez clases que rigen a las cinco actividades, comprobando la validación del usuario, la comunicación entre la aplicación móvil y el sistema embebido a través de los protocolos TCP y SSH, la descarga e instalación de las librerías, el acceso y funcionamiento del modo de referencia y de los modos de operación manual y automático, y la emulación del proceso industrial. Cuando se seleccionó a través del emulador el modo de operación automático, se comprobó la lógica de control por la activación y desactivación secuencial de manera autónoma de las electroválvulas y actuadores neumáticos, logrando con éxito el funcionamiento del proceso. Cuando se seleccionó el modo de operación manual, el usuario interactuó con las variables manipuladas (electroválvulas y actuadores), manteniendo la variable controlada, aplicando la lógica de control y logrando con éxito la operación del proceso. 
Además de las pruebas con el emulador, la aplicación móvil se instaló en los teléfonos móviles celulares Samsung Galaxy SIV mini y Moto G, y se realizaron pruebas de funcionamiento, en la figura 6 se aprecian los pantallazos de la interfaz de usuario. En las pruebas con los teléfonos celulares reales, se evaluaron los tres requerimientos no funcionales básicos del sistema, conocidos como estabilidad, portabilidad y conexión. La estabilidad de la aplicación se evidenció porque no ocurrieron errores (excepciones) en el tiempo de ejecución. La portabilidad se comprobó con la ejecución a satisfacción de la aplicación en los teléfonos móviles de marca Samsung y Motorola. La conexión fue correcta a través de la red de datos porque las solicitudes emitidas desde el teléfono fueron recibidas en todos los casos por el sistema embebido.

Cuando se ingresó al aplicativo MasterMobile, la interfaz de bienvenida estuvo a cargo de la actividad Master_home, para continuar a la actividad Master_login que tiene dos campos para insertar información (usuario y contraseña) y cuatro botones (Acceder, Modo de referencia, Ayuda y Salir), ver figura 6 . El botón Acceder validó el usuario y la contraseña como protocolo de seguridad. Con el botón Modo de referencia se conoció la función de las variables del proceso, sin tener control ni supervisión de las mismas. El botón de Ayuda mostró información relevante de la interfaz, facilitando la comprensión y toma de decisiones por parte del usuario, cuando interactuó con la aplicación. El botón Salir permitió al usuario abandonar la aplicación.

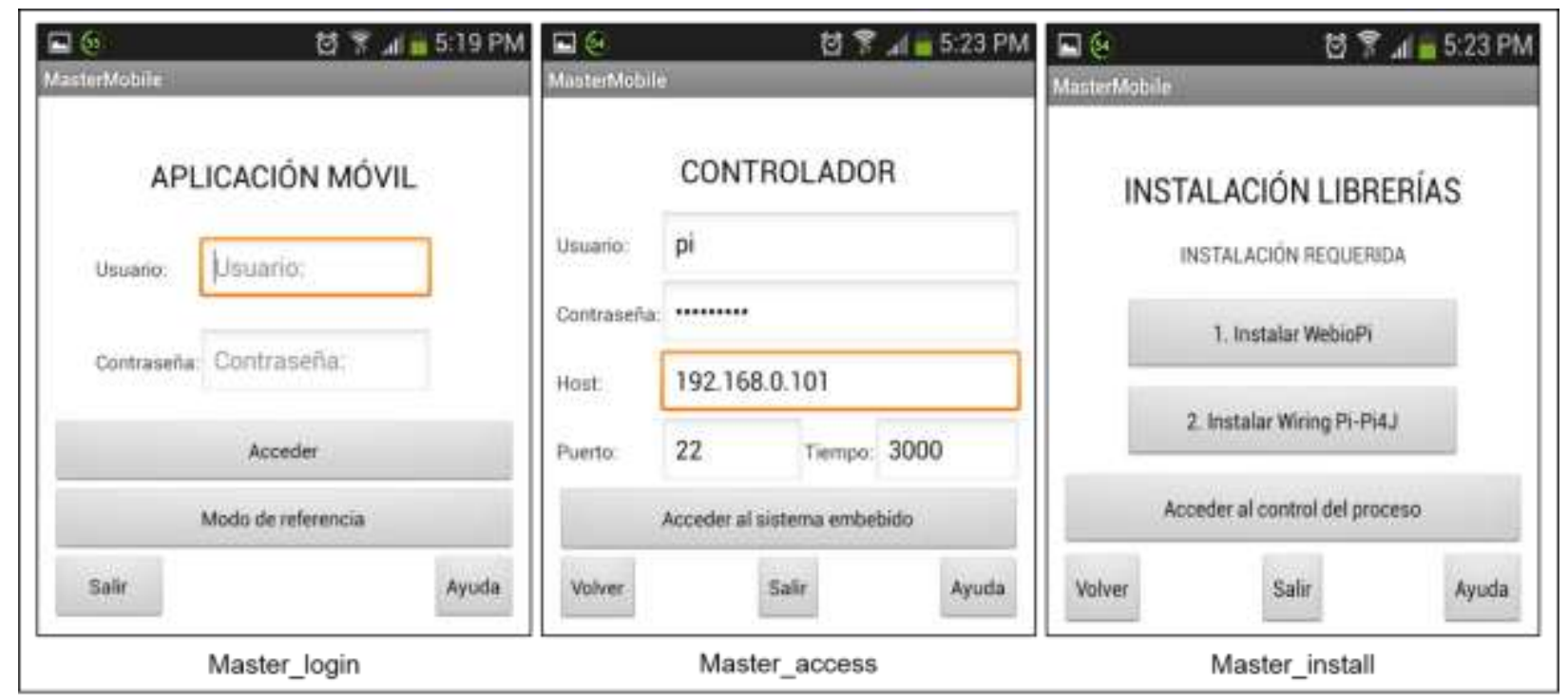

Fig. 6: Actividades Master_login, Master_access y Master_install

Cuando se seleccionó Acceder, la aplicación pasó a la clase Master_access (ver figura 6). En esta clase, el usuario digitó los datos, accediendo al sistema embebido, teniendo en cuenta que el usuario, la contraseña, el puerto y el tiempo estaban establecidos por defecto, pero con la posibilidad de cambiarse según requerimientos y políticas del desarrollador. De la misma manera, el usuario se regresó mediante el botón Volver, y consultó información mediante el botón de Ayuda. Cuando los datos se validaron correctamente, una notificación desplegó el mensaje de conexión exitosa y se accedió a la siguiente actividad. Cuando se validaron datos incorrectos, la notificación desplegó el mensaje de conexión fallida. Después de acceder al sistema embebido, el usuario que por primera vez ejecutó la aplicación en el dispositivo móvil, debió instalar las librerías WebIOPi, Wiring Pi y Pi4J, que fueron suministradas por la aplicación, y que fueron necesarias para interactuar con el sistema embebido. En la figura 6, se observa la implementación de esta actividad y es denominada Master_install.

La última actividad a la que se accedió como usuario se denomina Master_mobile (ver figura 7); esta actividad emuló el proceso industrial mediante una representación gráfica, centralizó el funcionamiento de las variables en el tablero de control, y permitió manipular las variables en los modos de operación manual y automático establecidos en la lógica de control. Además, en el modo de referencia se accedió a la información de las variables posicionando el cursor en la gráfica del proceso.

Cuando se seleccionó el modo de operación automático, se activó la secuencia de los actuadores de manera autónoma, las electroválvulas fueron manipuladas por el sistema embebido, siguiendo siempre con rigor la lógica de control. Cuando se seleccionó el modo de operación manual, éste permitió manipular las electroválvulas y cambiar sus estados, activando y desactivando los actuadores; además, se aplicaron secuencias de activación aleatorias para validar el algoritmo de la lógica de control del proceso, evidenciando que solo fue posible cumplir la secuencia establecida, para lo cual la aplicación orientó al usuario utilizando notificaciones. 


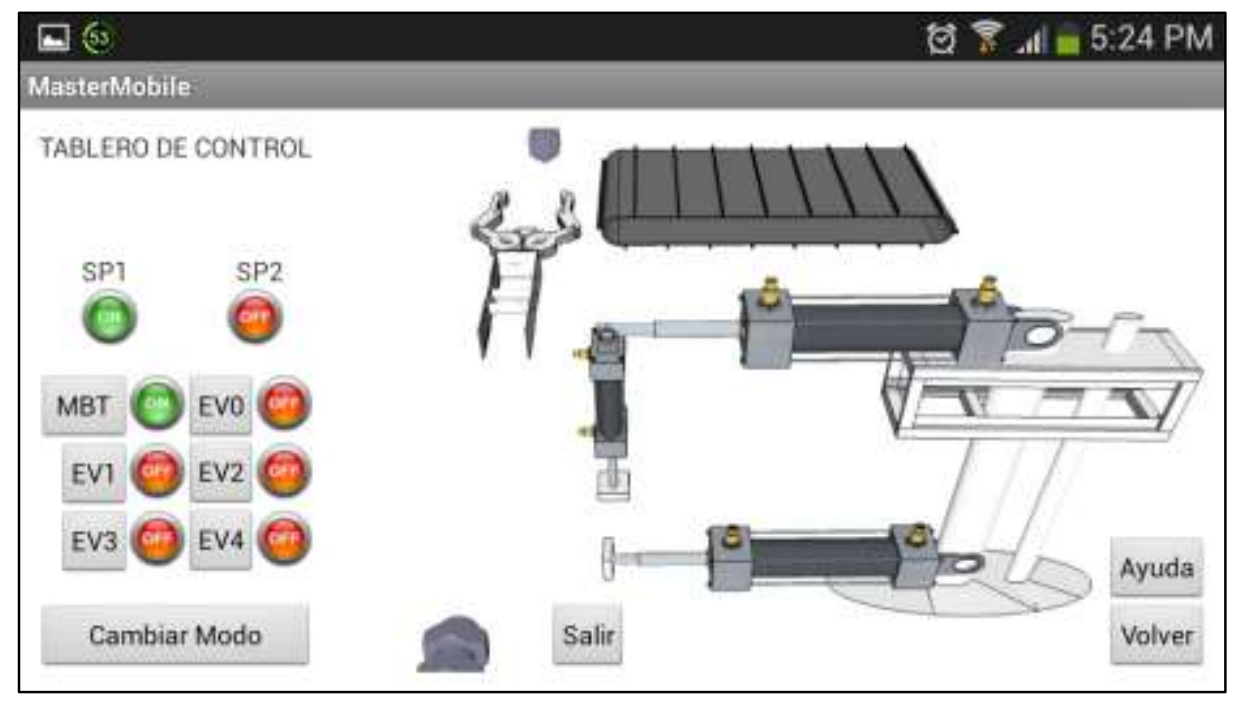

Fig. 7: Actividad Master_mobile

Finalmente, la aplicación móvil fue evaluada a través del potencial de éxito de las $6 \mathrm{M}$ 's y de la usabilidad. El potencial de éxito de las 6M's cuantificó seis atributos denominados movimiento (movement), momento (moment), yo (me), múltiples usuarios (multi-user), dinero (money) y máquinas (machines); mientras que la usabilidad cuantificó cinco atributos denominados facilidad de aprendizaje, eficiencia, recuerdo en el tiempo, manejo de errores y satisfacción. El instrumento aplicado en la evaluación incluyó once preguntas cerradas, y valoró el cumplimiento de cada atributo entre uno (en total desacuerdo) y cinco (en total acuerdo).

La cuantificación se realizó con una muestra de cuarenta personas con conocimientos en la utilización e implementación de tecnologías, de los cuales treinta fueron estudiantes de último semestre de ingeniería electrónica y diez ingenieros electrónicos. Este proceso consistió en la interacción de los cuarenta usuarios con la aplicación móvil en el escenario real, haciendo uso de los teléfonos celulares Samsung Galaxy SIV y Moto $\mathrm{G}$, a través de la red de datos privada; en donde cada persona a través del instrumento de evaluación, le asignó una puntuación entre uno y cinco a cada atributo, de acuerdo con el nivel de cumplimiento que experimentó, cuando interactuó con la aplicación. En la figura 8 se representa el valor promedio para cada atributo, obtenido con la media, porque el conjunto de datos para cada atributo no presentó valores atípicos.

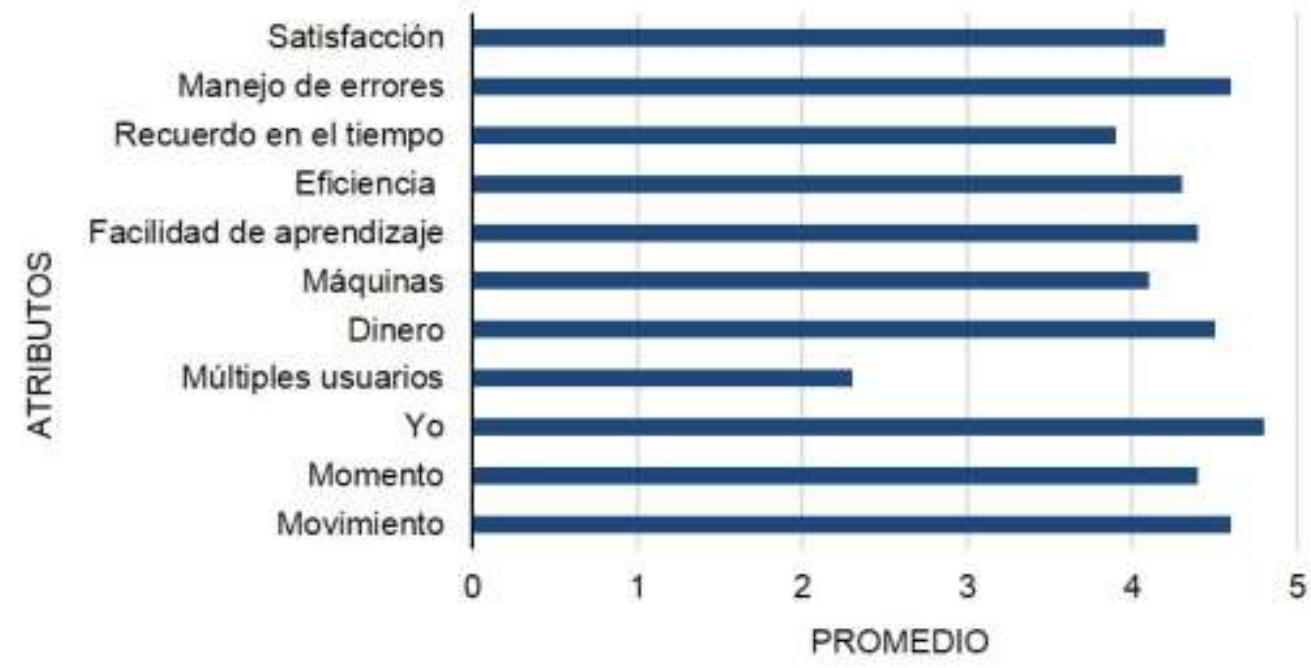

Fig. 8: Evaluación de atributos 6M's y usabilidad

De acuerdo con los resultados de evaluar la aplicación móvil se puede inferir que, la interfaz se ajustó a los requerimientos técnicos del proceso (atributo yo); permitió al operario desplazarse dentro de una zona de cobertura mientras gestionó remotamente y de manera controlada las variables del proceso (atributo movimiento); los algoritmos desarrollados no permitieron cometer errores en la operación de los modos manual y automático, y las notificaciones orientaron la interacción del usuario con la interfaz (atributo manejo de errores); evitó el uso de licencias, permitió al operario realizar tareas paralelas y utilizó la red no tarifada de área local (atributo dinero); supervisó y controló las variables del proceso, las veces que el 
operario requirió hacerlo (atributo momento); es intuitiva y, el modo de referencia y las ayudas orientaron de manera oportuna al operario (atributo facilidad de aprendizaje); estuvo sujeta a la confiabilidad de la red de área local (atributo eficiencia); cumplió con las expectativas de los usuarios (atributo satisfacción); posibilitó tecnológicamente la gestión remota del proceso (atributo máquinas); las notificaciones, el modo de referencia y las ayudas fortalecieron el proceso de aprendizaje (atributo recuerdo en el tiempo); y carece de un módulo de comunicación interno para los operarios del proceso industrial en la misma interfaz (atributo múltiples usuarios).

\section{CONCLUSIONES}

Los resultados estadísticos de evaluar los atributos de las 6M's y la usabilidad, en la metodología utilizada, sustentan que la aplicación móvil es fácil de manipular, es atractiva al usuario, es eficiente y presenta un manejo adecuado de errores, garantizando la usabilidad de la misma; además, que la aplicación tiene potencial de éxito para ser implementada a gran escala.

La conexión remota, en la arquitectura de red del sistema, mantuvo la integridad de la información, mediante el uso del protocolo y del programa de intérprete de órdenes segura, del método basado en expectativas, y del canal de comunicación seguro de java. Además, esta conexión garantizó que las peticiones realizadas desde los teléfonos inteligentes se ejecutaran con rigor en el sistema embebido, en todos los casos; y comprobó la posibilidad de implementar a la Raspberry Pi B como servidor.

La aplicación móvil para supervisar y controlar las variables del proceso industrial a través de los teléfonos inteligentes, no pretende reemplazar los sistemas SCADA tradicionales, solo busca ofrecer una alternativa para la gestión remota del estado de variables, en un mundo de alta movilidad, de escaso tiempo y que requiere el desarrollo de tareas en paralelo.

El empaquetamiento de las librerías en archivos comprimidos ejecutables para la aplicación, suministró seguridad porque el contenido se firmó digitalmente, disminuyó el tiempo de descarga del ejecutable porque éste se realizó en una sola transacción mediante el protocolo de transferencia de hipertexto, y redujo el contenido de información, contribuyendo a la portabilidad.

El uso de tecnologías de código abierto, evitó invertir recursos económicos en el pago de licencias, y exigió de un recurso humano con avanzados conocimientos en las áreas de programación, sistemas embebidos, control y telecomunicaciones. Además, demostró que un sistema embebido de plataforma independiente como la Raspberry puede ser utilizado para supervisar, controlar y adquirir datos, en un entorno industrial.

\section{REFERENCIAS}

Agusti, R., Bernardo, F., Casadevall, F., Ferrús, R., Pérez, J. y Sallent, O., LTE: nuevas tendencias en comunicaciones móviles, $1^{\text {a }}$ edición, 55-60, Fundación Vodafone España, Catalunya, España (2010)

Ahonen, T., Barret, J. y Golding, P. Services for UMTS, creating killer applications in 3G. West Sussex: John Wiley \& Sons, (2002)

Alarcón, A., Díaz, E., y Callejas M., Guía para la evaluación de la usabilidad en los Entornos Virtuales de Aprendizaje (EVA), doi: 10.4067/S0718-07642014000300016, Revista Información Tecnológica, (en línea), 25(3), 135-144 (2014)

Android Developers, Installing the Eclipse Plugin

http://developer.android.com/sdk/installing/installing-adt.html. Acceso: 14 de febrero (2014)

Beck, K., Beedle, M., Bennekum, A., et al., The Agile Alliance. Manifesto for Agile Software Development (2001), http://agilemanifesto.org/. Acceso: 1 de octubre (2012)

Breivold, H.P., Jansen, A., Sandstrom, K. y Crnkovic, I., Virtualize for Architecture Sustainability in Industrial Automation, Computational Science and Engineering (CSE), 2013 IEEE 16th International Conference on Computational Science and Engineering, pp.409,415, 3-5 Dec. (2013)

Camargo, L., Medina, B., y Gómez, J., Servicio de M-Comercio. Sistema de interacción entre un centro comercial y sus visitantes utilizando las tecnologías WAP y Bluetooth, doi: http://dx.doi.org/10.4067/S071833052013000100009, Revista Chilena de Ingeniería, (en línea), 21(1), 99-110 (2013) 
Cisco, Visual Networking Index Mobile Forecast Highlights, 2014-2019: Latin America - Device Growth Traffic Profiles, disponible en

http://www.cisco.com/c/dam/assets/sol/sp/vni/forecast_highlights_mobile/index.html. Acceso: 1 de marzo (2015)

Comisión Económica para América Latina y el Caribe (CEPAL). Estrategias TIC ante el desafío del cambio estructural en América Latina y el Caribe, Naciones Unidas, pp 7-10, Santiago de Chile, Chile (2013)

Eclipse Foundation open source community website, Eclipse RSS (2014), http://www.eclipse.org. Acceso: 14 de febrero (2014)

Gasca, M., Camargo, L. y Medina, B., Metodología para el desarrollo de aplicaciones móviles, doi: http://dx.doi.org/10.14483/udistrital.jour.tecnura.2014.2.a02, Revista Tecnura, (en línea), 18(40), 20-35 (2014)

Google Inc., Google Play (2015), https://play.google.com/store/apps. Acceso: 2 de febrero (2015)

Hurtado, L., Castrillón, O., y Olivar G., Una Metodología Automatizada para la Evaluación de Usabilidad de Interfaces de Supervisión Industrial, doi: 10.4067/S0718-07642013000400011, Revista Información Tecnológica, (en línea), 24(4), 95-104 (2013)

lacopino, P., Mobile handset data revenue will be driven by volume in emerging markets and value in developed countries, Analysys Mason, enero 6, 2014, disponible en http://www.analysysmason.com/AboutUs/News/Insight/mobile-handset-data-Jan2014-RDDG0/. Acceso: 15 de febrero (2015)

Mascheroni, M., Greiner, C., Dapozo, G. y Estayno, M., Automatización de la evaluación de la Usabilidad del Software, XV Workshop de investigadores en ciencias de la investigación, 572-575 (2013)

Medina, B., Castro, S. y Camargo, L., Tecnologías de código abierto para la gestión de un proceso industrial, Gerencia Tecnológica Informática, 14(38), 43-58 (2015)

OCDE/CEPAL (2011), Perspectivas Económicas de América Latina 2012: Transformación del Estado para el Desarrollo, OECD Publishing. http://dx.doi.org/10.1787/leo-2012-es. Acceso: 12 de Agosto (2014)

OPC Foundation, The Interoperability Standard for Industrial Automation (2015). http://www.opcfoundation.org. Acceso: 5 de junio (2015)

Oracle Technology Network, Oracle Technology Network for Java Developers (2014), http://www.oracle.com/technetwork/java/index.html. Acceso: 14 de febrero (2014)

Pi4J, The Pi4J Project (2014). http://pi4j.com. Acceso: 12 de marzo (2014)

Raspberry, Raspberry Pi (2014), http://www.raspberrypi.org. Acceso: 12 de marzo (2014)

Rivera, M.J., Camargo, L.L. y Medina, B., Servicio de mHealth para supervisar medidas corporales de glucosa y tensión arterial, doi: 10.1234/rielac.v35i2.239, Revista de Ingeniería Electrónica, Automática y Comunicaciones, (en línea), 35(2), 62-72 (2014)

Rodríguez, L. G., Prefacio, Lecciones aprendidas sobre adopción de TIC en PYMES: la experiencia del FOMIN/BID en América Latina, Fundación Telefónica Venezuela, pp 11-12, República Bolivariana de Venezuela, (2014)

UIT, Transformación de las telecomunicaciones, del pasado al futuro (2015), https://itunews.itu.int/Es/5914Del-pasado-al-futuro.note.aspx. Acceso: 3 de junio (2015)

Webiopi, Webiopi - Raspberry Pi Internet of Things framework - Google Project Hosting (2014). https://code.google.com/p/webiopi. Acceso: 12 de marzo (2014)

Wiring Pi, Wiring Pi (2014). http://wiringpi.com. Acceso: 12 de marzo (2014) 